\title{
A Deeper Look into the Ocular Lens
}

\author{
D.A. Scheiblin*, K.J. Czymmek*, Y. Wang*, and M.K. Duncan* \\ *Department of Biological Sciences, University of Delaware, Newark, DE 19716
}

The lens is an asymmetrical oblate spheroid composed of a monolayer of germinative cells (epithelial cells) that give rise to elongated, terminally differentiated cells (lens fiber cells) [1]. Cataracts, the clouding of the ocular lens, are the leading cause of blindness worldwide [2]. Since there are diverse etiologies for cataract including epithelial mesenchymal transition of the lens epithelium, disruption of fiber cell structure either associated or not with cytoskeletal alterations, and protein aggregation, determination of the precise structural alterations in cataractous lenses is crucial to understand the molecular basis of lens function and disease. However, the lens is a challenging structure to image since its high protein content and avascularity lead to poor fixative penetration. Furthermore, the high protein content makes it very difficult to obtain high quality sections, leads to non-specific binding of antibodies and can result in light scatter/autofluorescence [3]. In order to overcome these technical hurdles, we have developed improved techniques for examining both clear and cataractous lens, revealing both gross and ultrastructural details.

The majority of the lens actin cytoskeleton is cortical, and when intact stabilizes the structure of lens cells. Since it is difficult to obtain a detailed view of this structure in sectioned material, we developed a confocal method to visualize the actin cytoskeleton of the intact lens (Fig. 1A), which provided 3D structure of much of the lens without physical sectioning. These lenses were fixed in 4\% paraformaldehyde, washed in PBS with triton $\mathrm{x}-100$, incubated 2 days in phalloidin, washed and imaged. The lens epithelium is the most metabolically active region of the lens, however it is difficult to visualize in sectioned material since these cells have a very flat morphology. However, since the lens basement membrane (lens capsule) is relatively thick, it is possible to microdissect it with the attached epithelial cells from the lens. The lens capsule and epithelial cells then can be flat mounted on slides and prepared for confocal imaging using methods similar to those used for cultured cells (Fig 1B,C). Fiber cells are long, thin, transparent cells that allow light to pass through. If the structure of the cell is disrupted, light will be scattered resulting in impaired vision. Thus, key to understanding some eye pathologies are determination of the 3D structure of fiber cells (Fig. $1 \mathrm{D}, \mathrm{E})$. We plan to obtain improved detail matching the refractive index of mounting medium and lens tissue [4]. "Ball and sockets" are interdigitions along the membranes that allow for increased surface area on lens fiber cells to aid in the transport of ions (Fig. 1F). Lens fiber cells are laid down in radial cell columns in order to transport ions through gap junctions and pumps from one cell to another since the lens is an avascular tissue (Fig. 1G,H,I). Special challenges with the physical properties of the lens necessitate the creation of novel image preparation methodologies in order to probe the lens, its structure, and disease. We expect these same strategies can be modified and applied to other sample types with high refractive indices and/or protein concentrations.

\section{References}

[1] J. R. Kuszak et al., J. of Ultrastructure and Molecular structure research, 100, (1988) 60-74.

[2] A. Ramalingam et al. Cancer Research, 68 (2008) 1683-1690.

[3] N.A. Reed et al. Journal of Immunological Methods 253 (2001) 243-252.

[4] T. Staudt et al. Microscopy Research and Technique 70 (2007) 1-9.

[5] This research was supported by the National Eye Institute EY015279 and EY12221, NCRR grant S10 RR027273-01 and INBRE grant 2 P20 RR016472-10. 


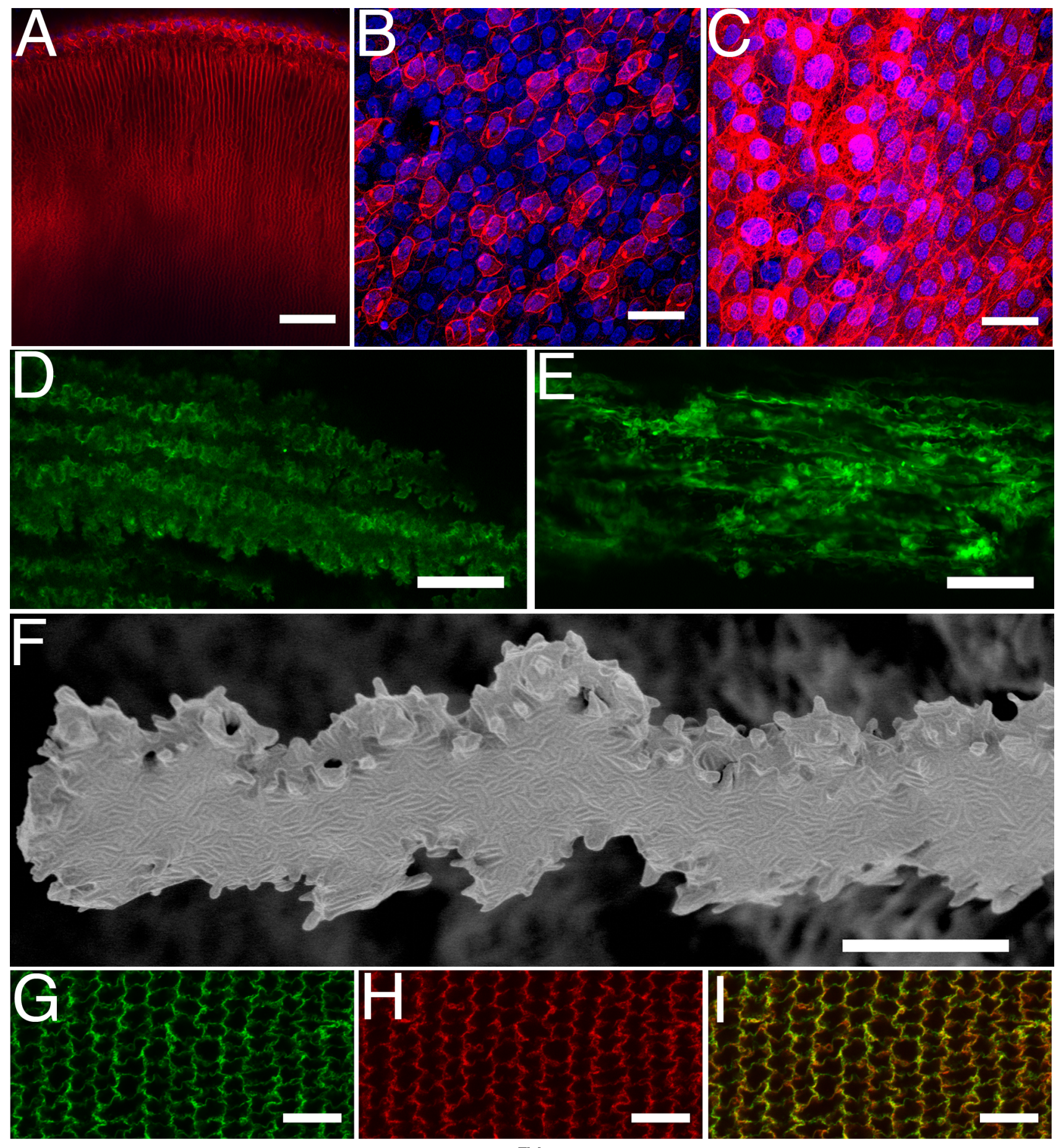

FIG.1. (A)A whole lens stained with Alexa Fluor ${ }^{\mathrm{TM}} 568 \mathrm{~nm}$ Phalloidin (red) and DRAQ5 (blue). Scale bar $=77 \mu \mathrm{m}$ (B) Wildtype and (C) cataractous epithelial whole mounts stained with Alexa Fluor ${ }^{\mathrm{TM}}$ $568 \mathrm{~nm}$ Phalloidin (red) and DRAQ5 (blue). Scale bar $=35 \mu \mathrm{m}$ (D) Whole wildtype fiber cells and (E) cataractous fiber cells stained with wheat germ agglutinin $488 \mathrm{~nm}$. Scale bar $=22 \mu \mathrm{m}$ (F) A wildtype fiber cell imaged on a field emission scanning electron microscope at 4000x. Scale bar= $10 \mu \mathrm{m}$ Wildtype lens fiber cells sectioned equatorially $(\mathrm{G})$ wheat germ agglutinin, $(\mathrm{H})$ phalloidin, and (I) merged. Scale bar $=12 \mu \mathrm{m}$ 